\title{
Fracture of electrodes in lithium-ion batteries caused by fast charging
}

\section{Citation}

Zhao, Kejie, Matt Pharr, Joost J. Vlassak, and Zhigang Suo. 2010. "Fracture of Electrodes in Lithium-Ion Batteries Caused by Fast Charging." Journal of Applied Physics 108 (7): 73517. https://doi.org/10.1063/1.3492617.

\section{Permanent link}

http://nrs.harvard.edu/urn-3:HUL.InstRepos:41461223

\section{Terms of Use}

This article was downloaded from Harvard University's DASH repository, and is made available under the terms and conditions applicable to Other Posted Material, as set forth at http:// nrs.harvard.edu/urn-3:HUL.InstRepos:dash.current.terms-of-use\#LAA

\section{Share Your Story}

The Harvard community has made this article openly available.

Please share how this access benefits you. Submit a story.

Accessibility 


\title{
Fracture of electrodes in lithium-ion batteries caused by fast charging
}

\author{
Kejie Zhao, Matt Pharr, Joost J. Vlassak, and Zhigang Suo ${ }^{\text {a) }}$ \\ School of Engineering and Applied Sciences and Kavli Institute, Harvard University, Cambridge, \\ Massachusetts 02138, USA
}

(Received 4 August 2010; accepted 13 August 2010; published online 8 October 2010)

\begin{abstract}
During charging or discharging of a lithium-ion battery, lithium is extracted from one electrode and inserted into the other. This extraction-insertion reaction causes the electrodes to deform. An electrode is often composed of small active particles in a matrix. If the battery is charged at a rate faster than lithium can homogenize in an active particle by diffusion, the inhomogeneous distribution of lithium results in stresses that may cause the particle to fracture. The distributions of lithium and stress in a $\mathrm{LiCoO}_{2}$ particle are calculated. The energy release rates are then calculated for the particle containing preexisting cracks. These calculations predict the critical rate of charging and size of the particle, below which fracture is averted. (c) 2010 American Institute of Physics. [doi:10.1063/1.3492617]
\end{abstract}

\section{INTRODUCTION}

Batteries are a key to the commercialization of clean and secure energy. ${ }^{1,2}$ Essential roles of batteries include leveling loads on power grids and storing energy from renewable sources. Furthermore, batteries are ubiquitous in all forms of electronics and transportation. For applications sensitive to weight and size, such as portable electronics and electric cars, the technology of choice is lithium-ion batteries. ${ }^{3}$

A lithium-ion battery contains an electrolyte and two electrodes. Each electrode is an atomic framework that hosts mobile lithium. During charging or discharging of the battery, lithium ions are extracted from one electrode, migrate through the electrolyte, and are then inserted into the other electrode. Meanwhile electrons flow from one electrode to the other through an external metallic wire. Extraction or insertion of lithium induces stresses in the electrodes that may cause fracture ${ }^{4}$ or morphological change. ${ }^{5}$ The loss of structural integrity may reduce electric conductance, causing the capacity of the battery to fade. ${ }^{6}$

Lithiation-induced damage is a bottleneck in developing batteries of high energy density. For example, the ability of silicon to absorb a large amount of lithium has motivated intense research ${ }^{7,8}$ but such absorption causes volumetric swelling of $\sim 400 \%$, leading to fracture. The mechanical failure has so far prevented silicon from serving as a viable electrode. Fracture has also been observed in commercial electrodes that undergo small deformation, such as $\mathrm{LiCoO}_{2}$, $\mathrm{LiMn}_{2} \mathrm{O}_{4}$, and $\mathrm{LiFePO}_{4}{ }^{4,9-13}$

Lithiation-induced deformation and stress have been studied in recent years. For example, Christensen and Newman ${ }^{14,15}$ calculated swelling and stress, Sastry and co-workers ${ }^{16,17}$ simulated the stress generation during lithiation under galvanostatic control, Cheng and Verbrugge ${ }^{18,19}$ calculated the strain energy under both potentiostatic and galvanostatic operations in spherical particles. Lithiation-

${ }^{a)}$ Electronic mail: suo@seas.harvard.edu. induced stress in silicon has been calculated. ${ }^{20,21}$ Several recent papers have studied lithiation-induced fracture by applying fracture mechanics. ${ }^{22-25}$

While a conceptual framework to analyze lithiationinduced deformation, stress, and fracture is emerging, limited work has been published that predicts fracture of practical systems by using actual material data. This paper attempts to predict fracture in a widely used material for cathode, $\mathrm{LiCoO}_{2}$. A commercial electrode often takes the form of active particles embedded in a binding matrix (Fig. 1). The distribution of lithium in the particle is inhomogeneous. The gradient of this inhomogeneity is large if the battery is charged at a rate faster than lithium can homogenize in the particle by diffusion. We calculate the distributions of lithium and stress in a $\mathrm{LiCoO}_{2}$ particle, and then calculate the energy release rates for the particle containing preexisting cracks. These calculations predict the critical rate of charging and size of the particle, below which fracture is averted.

\section{THEORY AND SCALING}

In a battery, the electrolyte conducts lithium ions but not electrons. When the battery discharges, the difference in the chemical potential of lithium in the two electrodes drives lithium ions to diffuse out of the anode, through the electrolyte, and into the cathode. To keep the electrodes electrically neutral, electrons flow from the anode to the cathode through

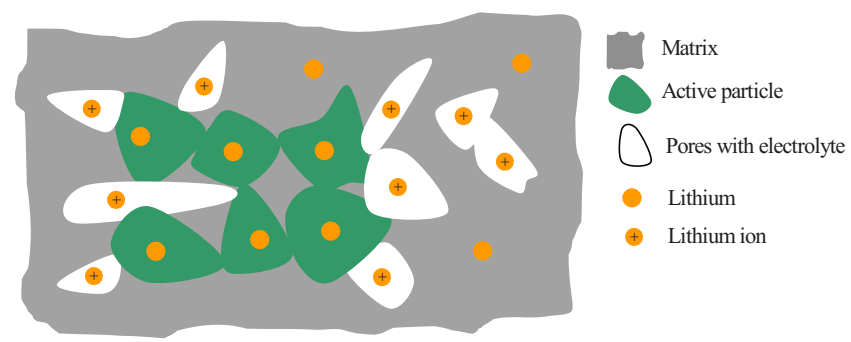

FIG. 1. (Color online) A schematic of the microstructure of the cathode. The cathode is composed of active particles, a matrix, and pores containing the electrolyte. 
(a)

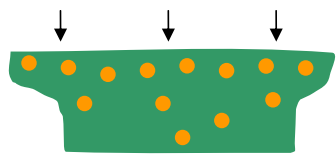

(b)

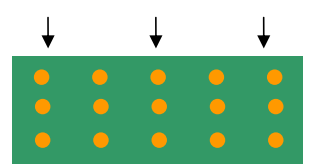

FIG. 2. (Color online) (a) When the rate of discharging is high, the distribution of lithium in the active particle is inhomogeneous, which causes a field of stress in the particle. (b) When the rate of discharging is low, the distribution of lithium in the particle is negligible. The arrows indicate the direction of lithium insertion.

the external metallic wire. Both the ionic and the electronic processes are reversed when the battery is charged by an external power source. As illustrated in Fig. 1, an electrode in commercial lithium-ion batteries is usually a composite, consisting of active particles, a compliant matrix composed of polymer binders and additives, and pores filled with the electrolyte. ${ }^{13,26}$ Migration of lithium in the electrolyte is fast, so that diffusion of lithium in the active particles limits the rate of charging and discharging. Because of the porosity of the composite and the compliance of the binder, stress in an active particle is often due primarily to the mismatch created by an inhomogeneous distribution of lithium within the particle.

The degree of the inhomogeneity depends on the competition between the rate of discharging and the rate of diffusion. Denote $\tau$ as the time to discharge the battery, $D$ a representative value of diffusivity of lithium in the particle, and $L$ the characteristic size of the particle. These quantities form a dimensionless group:

$$
\chi=\frac{L}{\sqrt{D \tau}} .
$$

The parameter $\chi$ characterizes the relative rate of discharging and diffusion. If $\chi$ is large, the battery is discharged at a rate faster than lithium can homogenize in the particle by diffusion. Consequently, lithium is crowded in the outer shell of the particle, causing a large stress in the particle [Fig. 2(a)]. By contrast, if $\chi$ is small, the battery is discharged at a rate slow enough for lithium in the particle to maintain nearly a homogeneous distribution. Consequently, lithiation causes the particle to swell or contract but the particle is nearly unstressed [Fig. 2(b)].

Cracklike flaws are assumed to preexist in the active particles. ${ }^{13}$ We ask if the lithiation-induced stress will cause any of the flaws to grow. The elastic energy in the particle reduces when a crack advances. The reduction in the elastic energy in the particle associated with the crack advancing a unit area defines the energy release rate $G$. Dimensional considerations dictate that the energy release rate should take the form

$$
G=Z E \varepsilon_{m}^{2} L,
$$

where $E$ is Young's modulus of the particle, $L$ a characteristic size of the particle, and $\varepsilon_{m}$ a characteristic mismatch strain, defined as

$$
\varepsilon_{m}=\frac{l_{1}-l_{0}}{l_{0}} .
$$

Here $l_{0}$ and $l_{1}$ are the lattice parameters in the initial state and in the fully lithiated state, respectively. At a given time, the distribution of the stress in the particle is determined by solving the diffusion equation, and the dimensionless coefficient $Z$ is determined by solving the elastic boundary-value problem. Once the geometry of the particle and the location of the crack are given, $Z$ can only vary with the length of the crack, the dimensionless parameter $\chi$, and time. We note this functional dependence in a normalized form:

$$
Z=f\left(\frac{a}{L}, \chi, \frac{t}{\tau}\right),
$$

where $a$ denotes the length of the crack. At a fixed value of $\chi$ and a fixed time, if the crack is very short, the elastic energy in the particle does not change appreciably when the crack grows, and the energy release rate is small. Likewise, when the crack is very long, the elastic energy is nearly fully relaxed because the crack introduces larger constraint-free area, and the energy release rate is also small. In between these two limits, the energy release rate reaches the maximum value $G_{\max }$ for a crack of a certain length. Let $\Gamma$ be the fracture energy of the particle. No preexisting flaws will advance if the maximum energy release rate is below the fracture energy of the particle

$$
G_{\max }<\Gamma \text {. }
$$

To ensure no preexisting flaws will advance, $G_{\max }$ indicates the energy release rate maximized for all configurations of the flaws and for all time. This approach has been used to analyze many systems, such as polycrystals, ${ }^{27}$ composites, ${ }^{28}$ and thin films. ${ }^{29}$

The comparison between the energy release rate and the fracture energy defines another dimensionless parameter

$$
\Lambda=\frac{E \varepsilon_{m}^{2} L}{\Gamma} .
$$

When $\Lambda$ is small, the elastic energy is insufficient to cause fracture. Therefore, a particle with small stiffness, small size, and large fracture energy is more resistant to fracture. This statement is consistent with recent experimental observation that the electrochemical cycling behavior is significantly improved if the size of active particles are small. ${ }^{30,31}$

The dimensionless groups $\chi$ and $\Lambda$ characterize the fracture behavior of the active particles. In the case of a highly inhomogeneous distribution of Li ions, to prevent fracture it is necessary to decrease the particle size, decrease the rate of discharging, and/or enhance the fracture energy. This concept is sketched schematically in Fig. 3 in terms of $\chi$ and $\Lambda$. The red line delineates an upper boundary to the safe regime in which no fracture occurs. 


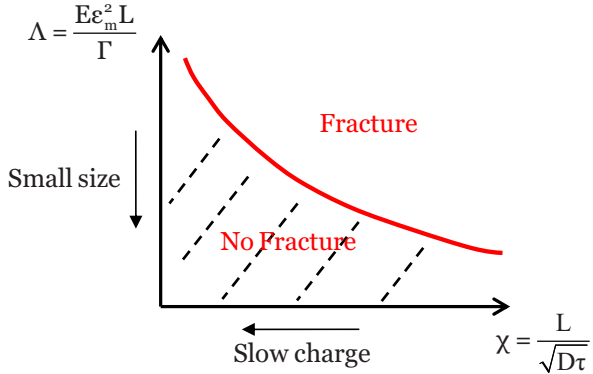

FIG. 3. (Color online) Criteria to avoid fracture of an electrode particle in terms of the nondimensional quantities $\Lambda$ and $\chi$.

The concentration of lithium in a particle is a timedependent field governed by the diffusion equation

$$
\frac{\partial c}{\partial t}=\left[D_{i j} c_{, j}\right]_{, i}
$$

where $c$ denotes the normalized lithium concentration in the host - the actual lithium concentration divided by the concentration of lithium in the fully lithiated state. The diffusivities in the crystal are in general anisotropic and are functions of the concentration, $D_{i j}(c)$. The concentration gradient is the primary driving force for diffusion. The effect of stress on diffusion has been explored in Ref. 16, and is found to be small. In this paper, we will neglect this small effect. Equa- tion (7), along with suitable initial and boundary conditions, determines the time-dependent concentration field in the particle.

The stress field is calculated by solving the elastic boundary-value problem. Since elastic deformation is much faster than atomic diffusion, mechanical equilibrium is established during lithium insertion. Therefore, the governing equations are the same as those in the theory of elasticity. Specifically, the strains $\varepsilon_{i j}$ relate to the displacements $u_{i}$ by

$$
\varepsilon_{i j}=\frac{1}{2}\left(u_{i, j}+u_{j, i}\right) .
$$

The stresses $\sigma_{i j}$ relate to the strains as

$$
\sigma_{i j}=C_{i j k l}\left(\varepsilon_{k l}-\varepsilon_{k l}^{L}\right),
$$

where $\varepsilon_{k l}^{L}$ are the insertion-induced strains and $C_{i j k l}$ represents the stiffness coefficients of the crystal. The stresses obey mechanical equilibrium, namely,

$$
\sigma_{i j, j}=0 .
$$

As mentioned before, the particle is embedded in a porous and compliant matrix, so that we will neglect any traction on the surface of the particle, namely,

$$
\sigma_{i j} n_{j}=0,
$$

where $n_{j}$ represents a unit vector normal to the particle surface. (a)

(c)
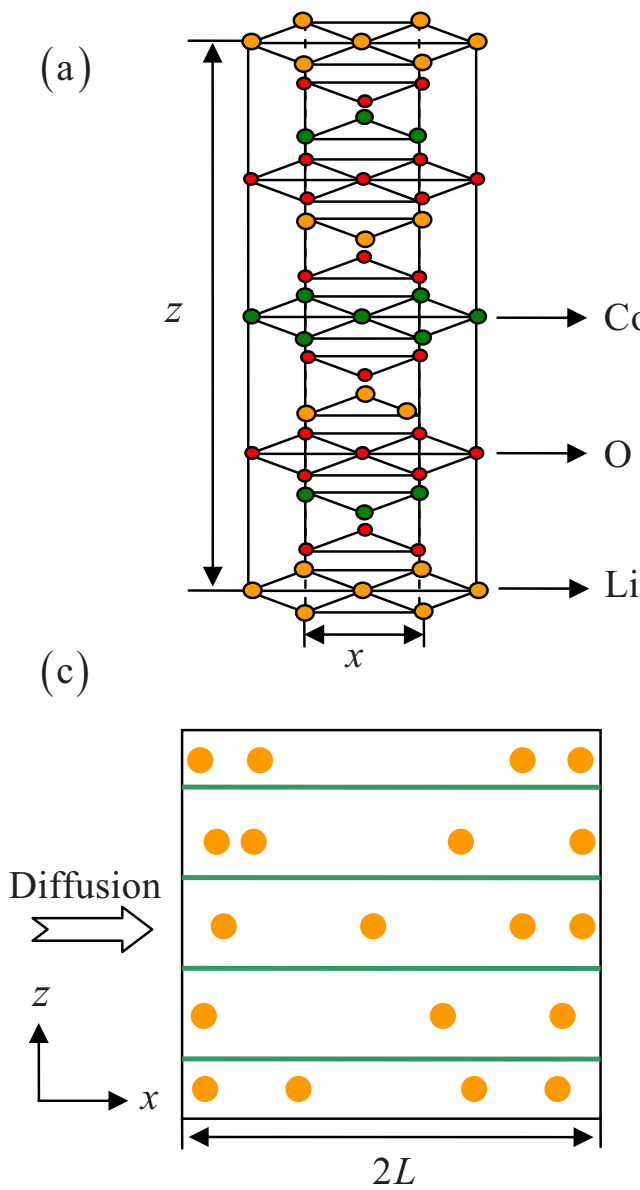

(b)

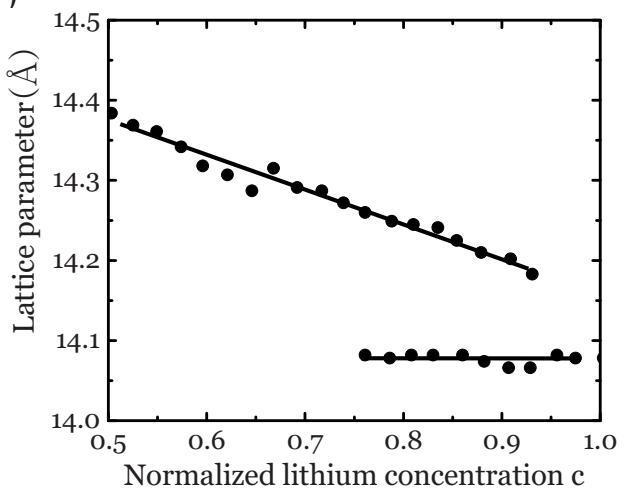

(d)

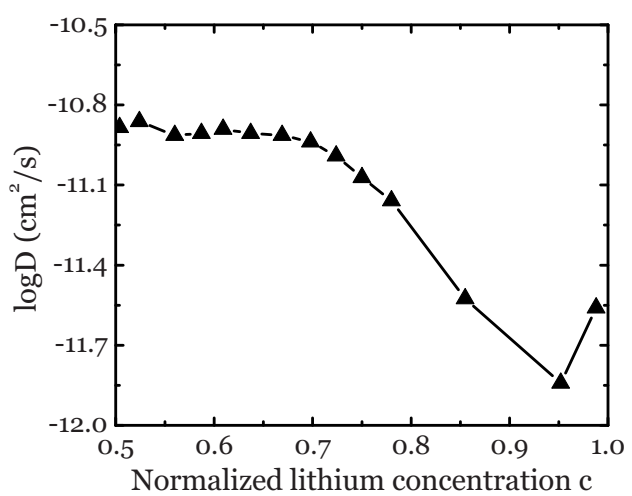

FIG. 4. (Color online) (a) The layered structure of $\mathrm{LiCoO}_{2}$. (b) Variation in lattice parameter along the $z$ axis with normalized lithium concentration (reproduced from Ref. 32). The lattice parameter along the $x$ axis is nearly a constant. (c) The cross section of the model. Lithium diffusion is along the $x$ axis, toward the center of the particle. (d) The lithium diffusivity as a function of the normalized lithium concentration (reproduced from Ref. 33). 
(a)

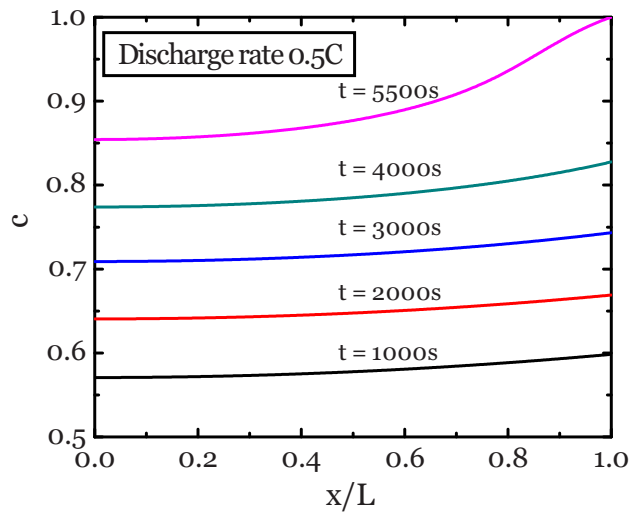

(b)

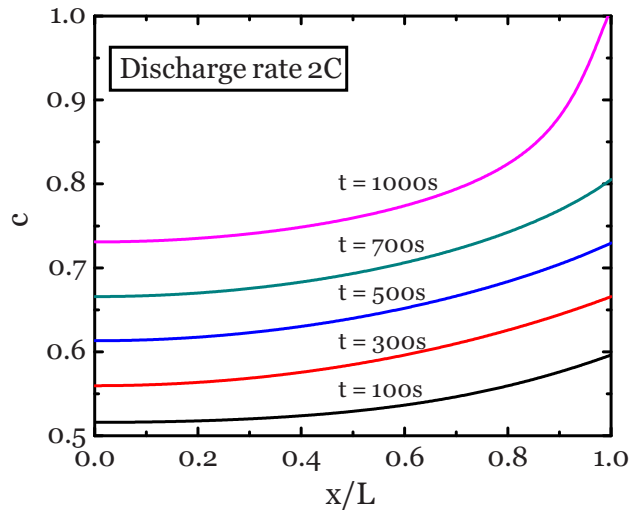

FIG. 5. (Color online) Lithium distribution with time for a $\mathrm{LiCoO}_{2}$ particle at (a) discharge rate of $0.5 C$, (b) discharge rate of $2 C$. Here $x / L=0$ represents the center of the particle, and $x / L=1$ represents the outside surface.

\section{NUMERICAL RESULTS FOR LICOO$_{2}$}

Following the above theory, we perform calculations for a $\mathrm{LiCoO}_{2}$ particle. As illustrated in Fig. 4(a), $\mathrm{LiCoO}_{2}$ has a layered crystalline structure, where oxygen ions form closepacked planes in an $A B C A B C$ sequence, and cobalt and lithium ions occupy alternating layers of octahedral sites between the oxygen layers. The oxygen ions cause repulsion between the neighboring layers. These oxygen layers are attracted to the lithium ions inserted between them. Thus, during discharge the insertion of lithium decreases the repulsion between the $\mathrm{CoO}_{2}$ sheets and leads to a contraction. ${ }^{32}$ The lattice parameter along the direction normal to the $\mathrm{CoO}_{2}$ sheets is shown as a function of lithium concentration in Fig. 4(b), which is reproduced from Ref. 32. The insertion of lithium also causes strains in the other directions of the crystal but these strains are much smaller, ${ }^{32}$ and will be neglected in our numerical simulation.

Diffusion of lithium is much faster in the planes between $\mathrm{CoO}_{2}$ sheets than in the direction normal to the $\mathrm{CoO}_{2}$ sheets. ${ }^{33}$ In the numerical simulation, we consider the diffusion in the planes between $\mathrm{CoO}_{2}$ sheets but neglect the diffusion normal to the sheets [Fig. 4(c)]. Experiments show that the diffusivity $D$ decreases one order of magnitude when the normalized lithium concentration increases from 0.5 to $1{ }^{33}$ The experimentally measured diffusivity as a function of the lithium concentration is reproduced in Fig. 4(d).

In practical applications, the size of an individual $\mathrm{LiCoO}_{2}$ particle in an electrode is on the order of microns or smaller. At such a small scale, the particle is often a single crystal or at most consists of a few grains. ${ }^{4}$ We assume that the particle is a single grain. In a commercial battery, $\mathrm{LiCoO}_{2}$ particles typically take an equiaxed shape. For simplicity, we assume that the particle deforms under the planestrain conditions, and has a square cross section of side $2 L$ [Fig. 4(c)]. The plane-strain assumption should not alter the scaling with respect to length and time.

As illustrated in Fig. 4(c), within the cross section, lithium only diffuses along the $x$ axis, between the $\mathrm{CoO}_{2}$ sheets. The diffusion of lithium in the particle is governed by Eq. (7). In practice, the working regime for lithium cobalt oxide is in the range of $0.5 \leq c \leq 1$. In the simulation we have allowed the diffusivity to vary with concentration, a dependence that is usually neglected in other works. The initial and boundary conditions are given by

$$
\begin{aligned}
& t=0 ; \quad c=0.5, \\
& x=0 ; t>0 \quad \frac{\partial c}{\partial x}=0, \\
& x=L ; t>0 \quad-D(c) \nabla c=\text { constant. }
\end{aligned}
$$

The constant in Eq. (12) is given by the discharge current density under galvanostatic (constant current) operation. Thus, in the simulation the discharge rate is controlled by changing the flux constant. Discharge is completed once the normalized concentration of lithium at the outside surface reaches a value of unity. The concentration profile of lithium inside the particle is obtained by solving the diffusion equation in COMSOL MULTIPHYSICS.

Figures 5(a) and 5(b) show the distributions of lithium at several times, at discharge rates of $0.5 C$ and $2 C$, respectively. Here the discharge rates are reported in the $C$-rate convention given by $C / \tau$, where the value of $\tau$ is calculated as the theoretical capacity $(\sim 140 \mathrm{mAh} / \mathrm{g})$ divided by the discharge current. It is evident from the figures that, in both cases, the lithium concentration gradient increases with discharge time. This behavior is a direct consequence of the decrease in effective diffusivity with increasing $\mathrm{Li}$ content [see Fig. 4(d)]. As the discharge rate increases from $0.5 C$ to of $2 C$, the lithium distribution inside the host particle becomes more inhomogeneous, in agreement with the schematics in Fig. 2.

This inhomogeneous distribution of Li results in a large deformation mismatch. The strain along the $z$ direction [see Fig. 4(a)] in the host particle is given by

$$
\varepsilon=\frac{l(c)-l_{0}}{l_{0}},
$$

where $l(c)$ is the lattice parameter in the direction normal to the $\mathrm{CoO}_{2}$ sheets at a given lithium concentration, $l_{0}$ is the lattice parameter at the initial normalized lithium concentration $c=0.5$. Here we use the experimental values for the $\mathrm{LiCoO}_{2}$ lattice parameter as a function of concentration to calculate the strain. ${ }^{32}$ The inhomogeneous distribution of lithium leads to a nonuniform strain field inside the particle and thus a stress field in the particle.

The elastic boundary-value problem is solved by using 
(a)

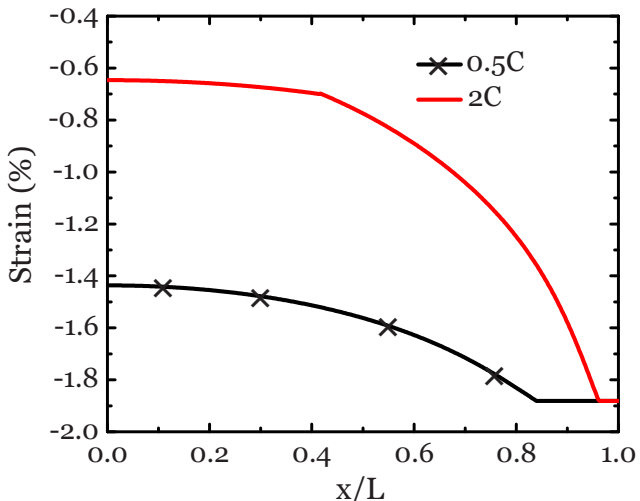

(b)

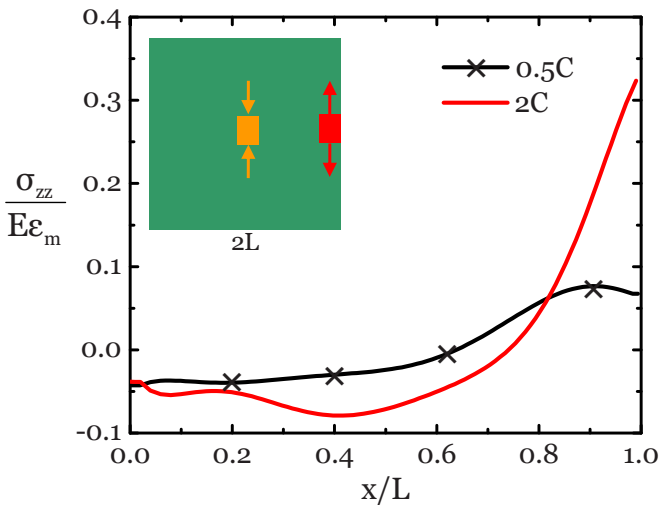

FIG. 6. (Color online) (a) Mismatch strain profile. (b) Normalized stress distribution at various discharge rates when the normalized lithium concentration at the outer surface reaches unity. The center of the particle is under compression, and the outside surface is under tension.

the finite element software ABAQUS. An orthotropic material model is employed with stiffness coefficients taken from atomistic simulations. ${ }^{34}$ The input strain field is simulated by imposing a thermal strain, equal to the concentration induced mismatch strain; the stress field is obtained as the output. In this configuration, the stress component $\sigma_{z z}$ is the tensile stress responsible for the crack propagation. Figures 6(a) and 6(b) plot the internal mismatch strains and the normalized stress distributions as a function of the dimensionless distance at discharge rates of $0.5 C$ and $2 C$, respectively. We represent $E$ as $C_{22}=375 \mathrm{GPa}$ used for the normalization. It can be seen that the outer shell (near $x / L=1$ ) of the particle is under tension, while the core (near $x / L=0$ ) is under compression during lithium insertion. The tensile stress in the outer shell may drive a preexisting crack to grow. The driving force is much larger for the faster discharge of rate $2 C$ because of the highly inhomogeneous distribution of lithium.

To calculate the energy release rate, a crack of length $a$ is assumed to preexist inside the particle. In the simulation, we fix the particle size and the location of the preexisting crack, and we vary the preexisting crack length and discharge rate. In each case, we use the stress distribution at the end of discharge. The $J$-integral is used to calculate the energy release rate. Figure 7 gives the normalized energy release rate dependence on the crack length to the particle size ratio, for discharge rates of $2 C, 4 C$, and $6 C$. The energy release rates are maximized at normalized lengths of 0.11 , 0.09 , and 0.07 , respectively.

As pointed out in Sec. II, no preexisting crack can ad-

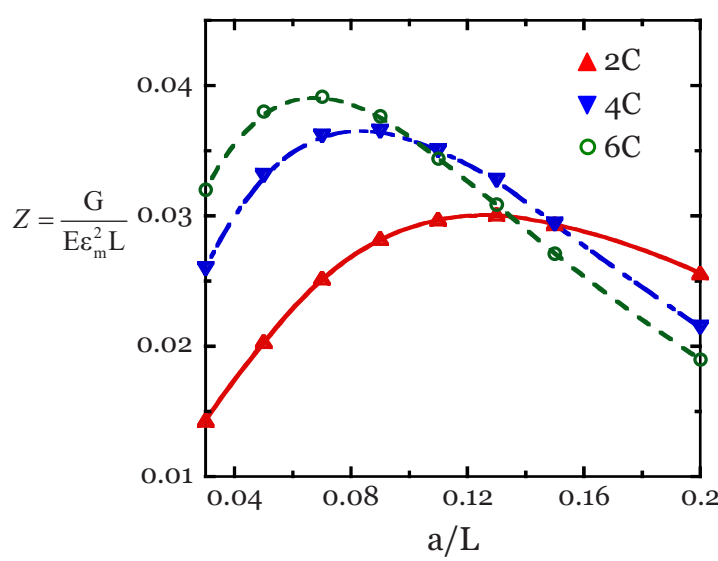

FIG. 7. (Color online) Energy release rate as a function of crack size at various discharge rates.

vance if the maximum energy release rate is smaller than the fracture energy. This condition defines a critical particle size

$$
L_{c}=\frac{\Gamma}{Z_{\max } E \varepsilon_{m}^{2}},
$$

where $Z$ is found from Fig. 7. When the particle is smaller than the critical value, no preexisting crack in the particle can advance. The critical particle size as a function of discharge rate is illustrated in Fig. 8. Here we have used approximate fracture energy of $1 \mathrm{~J} / \mathrm{m}^{2}$ for $\mathrm{LiCoO}_{2}{ }^{35}$ From the figure, decreasing the electrode particle size can effectively prevent fracture during fast charging.

Experimental observations of cracked $\mathrm{LiCoO}_{2}$ particles in the literature are limited. Crack damage is reported for an average particle size of $300-500 \mathrm{~nm}$ after 50 cycles. ${ }^{4}$ The model presented here shows that fracture can be prevented by decreasing the electrode particle size and/or discharge rate. To further test the accuracy of the model, more data for fracture of particles of different sizes and for a range of discharge rates are needed. Finally, it should be noted we used stiffness coefficients derived from atomistic simulations and estimated fracture energy to calculate the critical particle size. These values may vary with lithium concentration, ${ }^{36}$ and should be ascertained by further experiments.

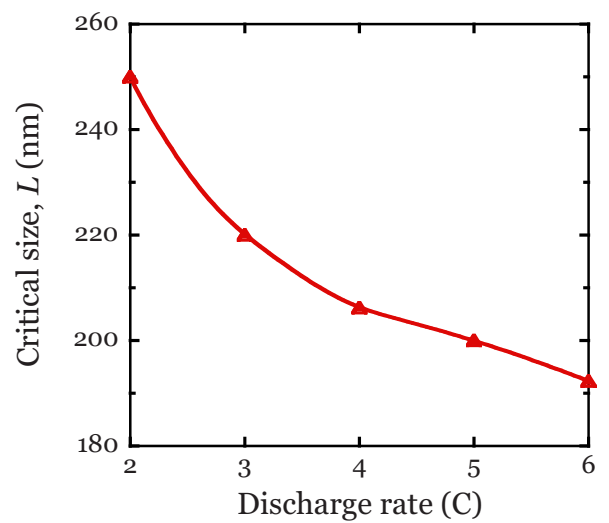

FIG. 8. (Color online) Critical $\mathrm{LiCoO}_{2}$ particle size to avoid crack propagation as a function of discharge rate. 


\section{CONCLUDING REMARKS}

Using a combination of diffusion kinetics and fracture mechanics, we have outlined a theory to study how material properties, particle size, and discharge rate affect fracture of electrodes in lithium-ion batteries. We characterize the discharge rate relative to diffusion rate by a dimensionless parameter $\chi$. We characterize the magnitude of the elastic energy relative to the fracture energy by a dimensionless parameter $\Lambda$. A "fracture map," demonstrating criteria for fracture, can be constructed in terms of the nondimensional parameters $\Lambda$ and $\chi$. To illustrate the theory, a numerical example of a $\mathrm{LiCoO}_{2}$ particle is presented. We calculate the distribution of lithium and stress at different discharge rates. We also calculate the energy release rates for preexisting flaws of different sizes. When the maximum energy release rate is smaller than the fracture energy, no pre-existing cracks can advance. This approach enables us to calculate the critical particle size and discharge rate necessary to avoid fracture.

\section{ACKNOWLEDGMENTS}

This work is supported by NSF through a grant on Lithium-ion Batteries (CMMI-1031161). Matt Pharr acknowledges the support of the Department of Defense (DoD) through the National Defense Science \& Engineering Graduate Fellowship (NDSEG) Program.

${ }^{1}$ Department of Energy, Basic Research Needs to Assure a Secure Energy Future, Washington, 2003.

${ }^{2}$ Department of Energy, Basic Research Needs for Electrical Energy Storage, Washington, 2007.

${ }^{3}$ G. A. Nazri and G. Pistoia, Lithium Batteries: Science and Technology (Kluwer Academic Publishers, Boston, MA, 2003).

${ }^{4}$ H. F. Wang, Y. I. Jang, B. Y. Huang, D. R. Sadoway, and Y. T. Chiang, J. Electrochem. Soc. 146, 473 (1999).

${ }^{5}$ C. K. Chan, H. L. Peng, G. Liu, K. McIlwrath, X. F. Zhang, R. A. Huggins, and Y. Cui, Nat. Nanotechnol. 3, 31 (2008).

${ }^{6}$ R. A. Huggins and W. D. Nix, Ionics 6, 57 (2000).
${ }^{7}$ U. Kasavajjula, C. S. Wang, and A. J. Appleby, J. Power Sources 163, 1003 (2007).

${ }^{8}$ V. A. Sethuraman, M. J. Chon, M. Shimshak, V. Srinivasan, and P. R. Guduru, J. Power Sources 195, 5062 (2010).

${ }^{9}$ M. G. Lazarraga, S. Mandal, J. Ibanez, J. M. Amarilla, and J. M. Rojo, J. Power Sources 115, 315 (2003).

${ }^{10}$ D. Y. Wang, X. D. Wu, Z. X. Wang, and L. Q. Chen, J. Power Sources 140, 125 (2005)

${ }^{11}$ G. Y. Chen, X. Y. Song, and T. J. Richardson, Electrochem. Solid-State Lett. 9, A295 (2006).

${ }^{12}$ H. Gabrisch, J. Wilcox, and M. M. Doeff, Electrochem. Solid-State Lett. 11, A25 (2008).

${ }^{13}$ J. R. Wilson, J. S. Cronin, S. A. Barnett, and S. J. Harris, J. Power Sources (in press).

${ }^{14}$ J. Christensen and J. Newman, J. Electrochem. Soc. 153, A1019 (2006).

${ }^{15}$ J. Christensen and J. Newman, J. Solid State Electrochem. 10, 293 (2006).

${ }^{16}$ X. C. Zhang, W. Shyy, and A. M. Sastry, J. Electrochem. Soc. 154, A910 (2007).

${ }^{17}$ X. C. Zhang, A. M. Sastry, and W. Shyy, J. Electrochem. Soc. 155, A542 (2008).

${ }^{18}$ Y. T. Cheng and M. W. Verbrugge, J. Power Sources 190, 453 (2009).

${ }^{19}$ Y. T. Cheng and M. W. Verbrugge, J. Appl. Phys. 104, 083521 (2008).

${ }^{20}$ R. Deshpande, Y. T. Cheng, and M. W. Verbrugge, J. Power Sources 195, 5081 (2010).

${ }^{21}$ S. Golmon, K. Maute, S.-H. Lee, and M. L. Dunn, Appl. Phys. Lett. 97, 033111 (2010)

${ }^{22}$ K. E. Aifantis, S. A. Hackney, and J. P. Dempsey, J. Power Sources 165, 874 (2007).

${ }^{23}$ Y. H. Hu, X. H. Zhao, and Z. G. Suo, J. Mater. Res. 25, 1007 (2010).

${ }^{24}$ T. K. Bhandakkar and H. J. Gao, Int. J. Solids Struct. 47, 1424 (2010).

${ }^{25}$ Y. T. Cheng and M. W. Verbrugge, J. Electrochem. Soc. 157, A508 (2010).

${ }^{26}$ M. S. Whittingham, MRS Bull. 33, 411 (2008).

${ }^{27}$ A. G. Evans, Acta Metall. 26, 1845 (1978).

${ }^{28}$ T. C. Lu, J. Yang, Z. Suo, A. G. Evans, R. Hecht, and R. Mehrabian, Acta Metall. Mater. 39, 1883 (1991).

${ }^{29}$ J. W. Hutchinson and Z. Suo, Adv. Appl. Mech. 29, 63 (1991).

${ }^{30}$ M. Jo, Y. S. Hong, J. Choo, and J. Cho, J. Electrochem. Soc. 156, A430 (2009).

${ }^{31}$ Y. Wang and G. Z. Cao, Adv. Mater. 20, 2251 (2008).

${ }^{32}$ J. N. Reimers and J. R. Dahn, J. Electrochem. Soc. 139, 2091 (1992).

${ }^{33}$ J. Xie, N. Imanishi, T. Matsumura, A. Hirano, Y. Takeda, and O. Yamamoto, Solid State Ionics 179, 362 (2008).

${ }^{34}$ F. X. Hart and J. B. Bates, J. Appl. Phys. 83, 7560 (1998).

${ }^{35}$ D. Kramer and G. Ceder, Chem. Mater. 21, 3799 (2009).

${ }^{36}$ V. B. Shenoy, P. Johari, and Y. Qi, J. Power Sources 195, 6825 (2010). 